\title{
Acute type A aortic dissection in the United Kingdom: Surgeon volume-outcome relation
}

\author{
Mohamad Bashir, MD, PhD, ${ }^{a}$ Amer Harky, MRCS, ${ }^{a}$ Matthew Fok, MBChB, ${ }^{b}$ Matthew Shaw, MS, ${ }^{c}$ \\ Graeme L. Hickey, PhD, ${ }^{\text {de, f }}$ Stuart W. Grant, PhD, ${ }^{\mathrm{e}, \mathrm{f}, \mathrm{g}}$ Rakesh Uppal, PhD, FRCS (CTh), ${ }^{\mathrm{a}}$ and \\ Aung Oo, FRCS (CTh) ${ }^{\mathrm{h}}$
}

\begin{abstract}
Objectives: Surgery for acute type A aortic dissection (ATAD) carries a high risk of operative mortality. We examined the surgeon volume-outcome relation with respect to in-hospital mortality for patients presenting with this pathology in the United Kingdom.

Method: Between April 2007 and March 2013, 1550 ATAD procedures were identified from the National Institute for Cardiovascular Outcomes Research database. A total of 249 responsible consultant cardiac surgeons from the United Kingdom recorded 1 or more of these procedures in their surgical activity over this period. We describe the patient population and mortality rates, focusing on the relationship between surgeon volume and in-hospital mortality.
\end{abstract}

Results: The mean annual volume of procedures per surgeon during the 6-year period ranged from 1 to 6.6 . The overall in-hospital mortality rate was $18.3 \%$ (283/1550). A mortality improvement at the $95 \%$ level was observed with a riskadjusted mean annual volume $>4.5$. Surgeons with a mean annual volume $<4$ over the study period had significantly higher in-hospital mortality rates in comparison with surgeons with a mean annual volume $\geq 4(19.3 \%$ vs $12.6 \% ; P=.015)$.

Conclusions: Patients with ATAD who are operated on by lower-volume surgeons experience higher levels of in-hospital mortality. Directing these patients to higher-volume surgeons may be a strategy to reduce in-hospital mortality. ( $\mathrm{J}$ Thorac Cardiovasc Surg 2017;154:398-406)

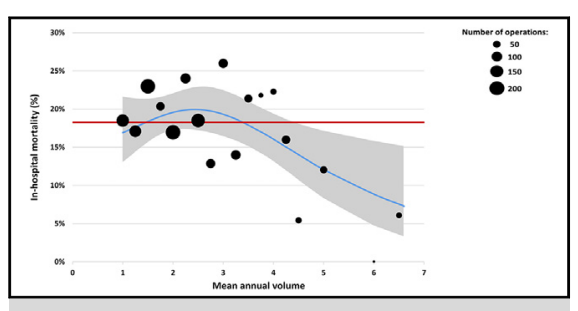

Survival in ATAD stratified by low- and high-volume surgeons.

\section{Central Message}

Lower-volume aortic surgeons experience higher in-hospital mortality for patients with ATAD. Concentrating ATAD repair to highvolume surgeons may be a strategy to reduce operative mortality.

\section{Perspective}

Analysis of the national United Kingdom database for all ATAD surgery revealed significantly higher in-hospital mortality in low-volume versus high-volume surgeons as well as 5-year survival. These results add to growing evidence that centralization of expertise and service provision for aortic dissection can increase survival.

See Editorial Commentary page 407.
Acute type A aortic dissection (ATAD) is a lethal condition and a cardiac surgical emergency. The incidence of aortic

\footnotetext{
From the a Department of Cardiac Surgery, Barts Heart Centre, St Bartholomew's Hospital, London; ' School of BUILT Environment, Liverpool John Moore University; ${ }^{\mathrm{c}}$ Research and Development and ${ }^{\mathrm{h}}$ Aortic Surgery, Liverpool Heart and Chest Hospital; ${ }^{\mathrm{d}}$ Department of Biostatistics, University of Liverpool, Liverpool; ${ }^{\mathrm{e}}$ University of Manchester, Manchester Academic Health Science Centre, Academic Surgery Unit, University Hospital of South Manchester, Department of Cardiothoracic Surgery, Manchester; ${ }^{\mathrm{f}}$ University College London, National Institute for Cardiovascular Outcomes Research (NICOR), London; and ${ }^{\mathrm{g}}$ Department of Cardiothoracic Surgery, Blackpool Victoria Hospital, Blackpool, United Kingdom.

Read at The American Association for Thoracic Surgery Aortic Symposium 2016, New York, New York, May 12, 2016.

Received for publication May 17, 2016; revisions received Jan 9, 2017; accepted for publication Feb 4, 2017; available ahead of print March 11, 2017.

Address for reprints: Mohamad Bashir, MD, PhD, Cardiothoracic Surgery, Barts Heart Centre, St Bartholomew's Hospital, West Smithfield, London EC1A 7BE, United Kingdom (E-mail: drmbashir@mail.com).

0022-5223/\$36.00

Copyright (c) 2017 by The American Association for Thoracic Surgery http://dx.doi.org/10.1016/j.jtcvs.2017.02.015
}

dissection is 30 to 43 per million population per year and this is incrementally increasing. ${ }^{1-3}$ Forty percent of patients with dissection are aged 60 to 74 but $27 \%$ are aged 17 to 59 years; thus, all ages are affected. ${ }^{4}$

In medically treated patients, mortality rates are $1 \%$ to $2 \%$ per hour after the initial event, with death due to coronary or other organ malperfusion, cardiac tamponade, or acute heart failure due to aortic regurgitation or aortic rupture. Emergency surgery can convert a 90\% mortality rate at 30 days to a $75 \%$ to $90 \%$ long-term survival rate.

Scanning this QR code will take you to the supplemental figure for this article. 

Abbreviations and Acronyms
AAD = acute aortic dissection
ATAD = acute type A aortic dissection
$\mathrm{CABG}=$ coronary artery bypass grafting
CCS = Canadian Cardiovascular Society
$\mathrm{CI}=$ confidence interval
$\mathrm{HR}=$ hazard ratio
IQR = interquartile range
IRAD = International Registry for Acute Aortic
MAV = mean annual case volume
NACSA $=$ National Adult Cardiac Surgery Audit
NICOR = National Institute for Cardiovascular Outcomes Research
NYHA $=$ New York Heart Association
ONS = Office for National Statistics
OR = odds ratio
SCTS = Society for Cardiothoracic Surgery
TAVI $=$ transcatheter aortic valve implantations

Mortality rates may vary. The International Registry for Acute Aortic Dissections (IRAD) ${ }^{5}$ and the United Kingdom Society for Cardiothoracic Surgery (SCTS) "Blue Book", have published operative mortality rates of $25.1 \%$ and $22.8 \%$, respectively. In contrast, the German registry GERAADA published their series with lower rates of $17 \%{ }^{7}$ This variation in reported mortality might be due to the volume-outcome relationship that has been at the center of debate and discussion. The Mount Sinai group using the Nationwide Inpatient Sample of North America reported that lower-volume surgeons and centers have approximately double the risk-adjusted mortality of patients undergoing repair by the highest-volume care providers. ${ }^{8}$

This study aims to report the national United Kingdom surgeon outcomes in the operated ATAD patient population and explore the relationship in this population between surgeon volume and adjusted in-hospital mortality.

\section{METHODS}

\section{National Institute for Cardiovascular Outcomes \\ Research Database}

Prospectively collected data were extracted from the National Institute for Cardiovascular Outcomes Research (NICOR) National Adult Cardiac Surgery Audit (NACSA) registry (version 4.1.2) on November 20, 2014, for all adult cardiac surgery procedures performed in the United Kingdom. As described elsewhere, reproducible cleaning algorithms were applied to the database. ${ }^{9}$ Briefly, duplicate records and nonadult cardiac surgery entries (including transcatheter aortic valve implantations [TAVIs]) were removed, transcriptional discrepancies harmonized, and clinical and temporal conflicts and extreme values corrected or removed. Data summaries are returned regularly to each unit for local validation as part of the NACSA in the United Kingdom. ${ }^{10}$

For this study, records were included that corresponded to the following criteria: procedure on 1 or more of the root, ascending, or arch aortic segments with a recorded pathology of "Acute Dissection" that were performed in England and Wales between April 1, 2007, and March 31, 2013. Records missing responsible consultant cardiac surgeon data (recorded in the form of General Medical Council registration number) were excluded.

\section{Baseline and Operative Variables}

For each procedure, data are recorded on patient characteristics, comorbidities, surgical team, intraoperative factors, and postoperative outcomes. For this study, we extracted data on patient age at the time of procedure (years), gender, body mass index (BMI, defined as weight $[\mathrm{kg}] / \mathrm{height}^{2}$ $\left.\left[\mathrm{m}^{2}\right]\right)$, Canadian Cardiovascular Society (CCS) angina class, dyspnea (dichotomized as New York Heart Association [NYHA] grade < III and NYHA grade $\geq$ III), recent myocardial infarction (defined as within 90 days of surgery), history of major cardiac surgery, diabetes (diet or insulin controlled), smoking status, history of hypertension, serum creatinine $>200 \mu \mathrm{mol} / \mathrm{L}$, history of renal dysfunction, history of pulmonary disease, history of neurological dysfunction, extracardiac arteriopathy, preoperative heart rhythm, left ventricular ejection fraction (classified as $<30 \%, 30 \%$ $50 \%$, and $>50 \%$ ), intravenous inotropes before anesthesia, preoperative ventilation, preoperative cardiogenic shock, operative urgency, concomitant coronary artery bypass grafting (CABG) and valve procedures, cardiopulmonary bypass time, aortic cross-clamp time, and circulatory arrest time.

Administrative data also were also extracted, including patient admission, procedure and discharge dates, responsible consultant cardiac surgeon, and anonymized hospital identifier. Further details of variable definitions are available at: http://www.ucl.ac.uk/nicor/audits/ adultcardiac/datasets.

\section{Outcomes}

The primary outcome for this study was in-hospital mortality, defined as death due to any cause during admission to the base hospital for cardiac surgery. The secondary outcome was mid-term mortality followed up to 5 years. Follow-up data up until the point of discharge was collected by the NACSA clinical registry system and postdischarge survival data were collected by linking the records via patient NHS numbers to the Office for National Statistics (ONS) death registry, which records all deaths in England and Wales. The final date of follow-up was July 30, 2013. Data on cause of death were unavailable. An attempt to back-fill missing inhospital mortality data was made by record linkage to the ONS registry before applying the extraction criteria.

\section{Case Volumes}

For analytical purposes, case volumes are presented both continuously and categorically. In the continuous analysis, the mean annual case volume (MAV) was calculated. This was achieved by taking the total number of procedures for each surgeon and dividing this by the number of years in which they contributed data to the registry. In the categorical analysis, the case volume was stratified into 2 groups (surgeon MAV of ATAD procedures $<4$ and $\geq 4$ over the study period). The cut point was selected as being clinically meaningful after the introductory analysis showed it to be the approximate inflection point for improved mortality.

\section{Statistical Analysis}

Categorical and dichotomous variables are summarized as absolute number and percentage. Non-normally distributed continuous data are summarized as median and interquartile range (IQR). The prevalence of missing data in the registry for baseline and operative measurements, as well as in-hospital mortality, are reported. Due to the relatively low number of missing data items for most of the variables, categorical variables with missing data were imputed with the baseline category and continuous variables were imputed with the mean value before calculations were performed. 
Where categorical comparisons are made between groups the $\chi^{2}$ test was used; for similar comparisons between continuous variables, the Wilcoxon rank-sum test was used.

To quantify the relationship between MAV and in-hospital mortality we performed 3 separate multivariable regression analyses. First, an initial balancing score was fitted for each patient using a mixed-effects linear regression model. The dependent continuous variable used was a log transformation of the surgeon MAV, with random intercepts for each hospital, and the following patient variables were entered as independent variables: age, gender, BMI, smoking, renal failure, hypertension, pulmonary disease, neurological disease, neurological dysfunction, peripheral vascular disease, recent myocardial infarction, unstable angina, arrhythmia, NYHA class, previous cardiac surgery, diabetes, ejection fraction, cardiogenic shock, preoperative ventilation, operative urgency, cardiopulmonary bypass time, circulatory arrest time, surgery on the aortic arch, and concomitant procedures. The volume-outcome relationship was then assessed by plotting estimated restricted cubic spline regression functions with 3 knots between the surgeon MAV and in-hospital mortality; these were then adjusted for patient case mix by entering the balancing score into the regression model. The spline estimates were based on a standard, fixed effects logistic regression model. The balancing score therefore acts in a similar way to a propensity score, ${ }^{11,12}$ but is generalized beyond a dichotomous treatment assignment.

Second, to examine the contribution of hospital volume to outcome, a logistic regression model for in-hospital mortality was fitted, including random intercepts for each hospital, with surgeon and hospital MAV entered into the model as continuous variables, along with the independent demographic and procedural variables listed previously, interactional terms between hospital and surgeon MAV were also explored.

Third, a similar approach was taken to assessing the contribution of MAV to mid-term mortality. Cox proportional hazards models were fitted, again with random intercepts for each hospital and with surgeon and hospital MAV entered into the model as continuous variables, along with the independent demographic and procedural variables listed previously. To demonstrate any nonproportional effects of early mortality, 2 models were fitted, one with a start time of the procedure date and the second with a start time of 90 days postprocedure.

To evaluate the categorical difference in volume, Kaplan-Meier charts were used to plot the actuarial 5-year survival, incorporating a landmark cutoff of 90 days in which the groupwise mortality rates were rebased to zero. The log-rank test was used to assess the equivalence of death rates between groups in both phases of the analysis.

Statistical analyses were performed with SAS version 9.3 (SAS Institute, Cary, NC). In all cases, $P<.05$ was considered statistically significant.

\section{RESULTS}

\section{Characteristics of the Study Population}

A total of 1632 patients were identified from the NACSA database as having had surgery for ATAD during the time period. Patients who had surgery on the descending and/ or thoracoabdominal segments of the aorta $(n=63$; $3.9 \%)$, and $19(1.2 \%)$ records that lacked responsible clinician data were excluded from the analysis. The 1550 that remained were included in the study analysis dataset; however, $162(10.5 \%)$ records lacked follow-up mortality data and are excluded from that element of the analysis.

The 1550 patients were admitted to 41 different hospitals throughout England and Wales and were under the care of 249 different consultant cardiac surgeons. The mean surgeon MAV was $2.6(\mathrm{SD}=1.2$; median $[\mathrm{IQR}]=2.3$ [1.63.3]), with $199(79.9 \%)$ of 249 surgeons performing fewer than 10 procedures overall. A total of 41 surgeons performed a single ATAD procedure. The highest number of procedures performed by a single surgeon during the study period was 33. The mean hospital MAV was $9.6(\mathrm{SD}=4.6$; median $[\mathrm{IQR}]=8.7[6.0-13.2])$. The highest number of procedures performed by a single hospital during the study period was 107.

Preoperative and operative differences between the 2 categorical groups are shown in Tables 1 and 2. Surgeons in the lower MAV group were more likely to operate on patients who had a recent myocardial infarction, and less likely to operate on patients with a history of pulmonary disease or patients who required surgery on the aortic arch. Surgeons in the lower MAV group also reported significantly longer circulatory arrest times.

\section{In-Hospital Mortality and Case Volume}

The overall in-hospital mortality rate for all ATAD patients was $18.3 \%$ (283 patients). Figure 1 plots the observed in-hospital mortality against the adjusted surgeon MAV. Somewhat counterintuitively, the curve begins below the national mean rate at the lowest volumes then rises and peaks between 2 and 3 procedures per year, before decreasing in an approximate linear trend in highervolume surgeons. Significant in-hospital mortality improvements can be observed beyond a surgeon MAV of 4.0 to 4.5 .

The unadjusted in-hospital mortality rate decreased from $19.3 \%$ in the group of surgeons who had an MAV $<4$ during the study period to $12.6 \%$ in the group who had an MAV $\geq 4 ; P=.015$ (Table 2). Figure 2, $A$, illustrates the groupwise trends in 5-year follow-up mortality rates, including a landmark rebasing at 90 days. The early difference in mortality rates is significant at the .05 level (log-rank test $P$ value $=.028$ ); however, this difference is not sustained in the second era, from 90 days to 5 years $(P=.97)$ (Figures E1 and 2, B, are details from Figure 2, $A$, which charts 5-year and 90-day mortality, respectively; they are included for clarity only).

The logistic regression model shown in Table 3 demonstrates a similar in-hospital mortality advantage for surgeons who perform a greater number of operations, after adjustment for case mix and hospital volume. Increasing surgeon MAV (assessed as a continuous variable) was associated with a significant reduction to in-hospital mortality (adjusted odds ratio $[\mathrm{OR}]=0.853 ; 95 \%$ confidence interval [CI] $0.733-0.992 ; P=.039)$. Other associated variables were increasing age, previous cardiac surgery, peripheral vascular disease, left ventricular ejection fraction $<30 \%$, cardiogenic shock, salvage priority, concomitant CABG procedure, and increasing cardiopulmonary bypass time. Hospital MAV was not associated with a difference in inhospital mortality (adjusted OR $=1.005 ; 95 \%$ CI $0.956-$ $1.057 ; P=.84)$. 
TABLE 1. Patient characteristics, stratified by surgeon MAV group

\begin{tabular}{|c|c|c|c|c|}
\hline Preoperative patient characteristics & $\begin{array}{c}\text { Mean annual volume }<4, \\
n=1319\end{array}$ & $\begin{array}{c}\text { Mean annual volume } \geq 4, \\
n=231\end{array}$ & $\boldsymbol{P}$ & Missing data \\
\hline Age at procedure, $y$ & $63(52,72)$ & $64(53,74)$ & .10 & $0(0)$ \\
\hline Female gender & $425(32.2)$ & $69(29.9)$ & .48 & $0(0)$ \\
\hline Body mass index, $\mathrm{kg} / \mathrm{m}^{2}$ & $26.4(23.9,29.7)$ & $26.4(24.2,29.0)$ & .54 & $48(3.5)$ \\
\hline Angina class IV & $199(15.1)$ & $39(16.9)$ & .48 & $17(1.1)$ \\
\hline NYHA class $\geq$ III & $354(26.8)$ & $68(29.4)$ & .41 & $23(1.5)$ \\
\hline Previous Q-wave MI & $130(9.9)$ & $18(7.8)$ & .32 & $14(0.9)$ \\
\hline Recent MI (within $90 \mathrm{~d}$ ) & $83(6.3)$ & $4(1.7)$ & .006 & $10(0.7)$ \\
\hline Previous PCI & $44(3.3)$ & $6(2.6)$ & .56 & $39(2.5)$ \\
\hline Previous cardiac surgery & $75(5.7)$ & $20(8.7)$ & .08 & $127(8.2)$ \\
\hline Diabetes (diet or insulin controlled) & $62(4.7)$ & $6(2.6)$ & .15 & $13(0.8)$ \\
\hline Current smoker & $243(18.4)$ & $34(14.7)$ & .18 & $64(4.1)$ \\
\hline History of hypertension & $905(68.6)$ & $167(72.3)$ & .26 & $27(1.7)$ \\
\hline Creatinine $>200 \mu \mathrm{mol} / \mathrm{L}$ & $67(5.1)$ & $16(6.9)$ & .25 & $115(7.4)$ \\
\hline History of renal dysfunction & $32(2.4)$ & $8(3.5)$ & .36 & $71(4.6)$ \\
\hline History of pulmonary disease & $134(10.2)$ & $34(14.7)$ & .04 & $9(0.6)$ \\
\hline History of neurological disease & $130(9.9)$ & $29(12.6)$ & .21 & $23(1.5)$ \\
\hline History of neurological dysfunction & $93(7.1)$ & $20(8.7)$ & .39 & $15(1.0)$ \\
\hline Peripheral vascular disease & $259(19.6)$ & $47(20.4)$ & .80 & $16(1.0)$ \\
\hline Nonsinus heart rhythm & $120(9.1)$ & $18(7.8)$ & .52 & $99(6.4)$ \\
\hline Triple vessel disease & $29(2.2)$ & $5(2.2)$ & .97 & $338(21.8)$ \\
\hline Left ventricular ejection fraction $30 \%-50 \%$ & $248(18.8)$ & $39(16.9)$ & .49 & $31(2.0)$ \\
\hline Left ventricular ejection fraction $<30 \%$ & $50(3.8)$ & $7(3.0)$ & .57 & $31(2.0)$ \\
\hline Intravenous nitrates or any heparin & $200(15.2)$ & $29(12.6)$ & .30 & $6(0.4)$ \\
\hline Intravenous inotropes before anesthesia & $100(7.6)$ & $10(4.3)$ & .08 & $10(0.7)$ \\
\hline Preoperative ventilation & $77(5.8)$ & $12(5.2)$ & .70 & $15(1.0)$ \\
\hline Preoperative cardiogenic shock & $228(17.3)$ & $29(12.6)$ & .07 & $14(0.9)$ \\
\hline
\end{tabular}

Continuous variables shown as median (25th percentile, 75 th percentile); categorical variables shown as frequency ( $\%)$. NYHA, New York Heart Association; $M I$, myocardial infarction; $P C I$, percutaneous coronary intervention.

Figure 3 includes 4 charts that show the interaction between surgeon and hospital MAV with regard to inhospital mortality. The predicted probabilities of inhospital mortality over the range of surgeon MAV are shown for the 20th, 40th, 60th, and 80th percentiles of hospital MAV (which are, respectively, 5.8, 7.0, 10.2, and 14.3 cases per year). Visual inspection of these allows us to infer that there are no substantial differences in the relationship between surgeon MAV and in-hospital mortality as hospital MAV increases. The associated interaction test $P$ value $=.88$.

\section{Follow-up Mortality and Case Volume}

The results of the 2 Cox proportional hazard models are shown in Table 4. In the "Start time = procedure date" model, higher surgeon MAV (assessed as a continuous variable) was again associated with a significant reduction in death (adjusted hazard ratio $[\mathrm{HR}]=0.882 ; 95 \% \mathrm{CI}$ $0.801-0.972 ; P=.011)$. Other variables that were associated with a greater hazard of death were increasing age, left ventricular ejection fraction $<30 \%$, cardiogenic shock, salvage priority, surgery on the aortic arch, concomitant CABG procedure and increasing cardiopulmonary bypass time. Hospital MAV was not significantly associated with a difference in death (adjusted HR $=1.029 ; 95 \% \mathrm{CI}$ $1.000-1.059 ; P=.050)$.

In the "Start time $=90$ days postprocedure" model, higher surgeon MAV was not associated with a significant reduction in death (adjusted HR $=0.920 ; 95 \%$ CI 0.779 $1.088 ; P=.33$ ). Other variables that were associated with a greater hazard of death were increasing age and left ventricular ejection fraction $<30 \%$. Hospital MAV was not significantly associated with a difference in death (adjusted $\mathrm{HR}=1.020 ; 95 \% \mathrm{CI} 0.972-1.072 ; P=.42)$, suggesting that the significant effect observed in the "Start time $=$ procedure date" model is both nonproportional and also greatly reliant on the large early mortality burden.

\section{DISCUSSION}

It has been shown that of every 1000 emergency department patients presenting with acute back, chest, or abdominal pain, 3 patients with ATAD are diagnosed. ${ }^{13}$ This is a fatal condition with a dire prognosis unless the patient receives immediate surgical intervention. The IRAD has published outcomes from multiple centers worldwide, with an average in-hospital mortality 
TABLE 2. Operative characteristics and in-hospital mortality, stratified by surgeon MAV group

\begin{tabular}{|c|c|c|c|c|}
\hline $\begin{array}{c}\text { Operative characteristics and } \\
\text { in-hospital mortality }\end{array}$ & $\begin{array}{l}\text { Mean annual volume }<4, \\
n=1319\end{array}$ & $\begin{array}{c}\text { Mean annual volume } \geq \mathbf{4}, \\
n=231\end{array}$ & $\boldsymbol{P}$ & Missing data \\
\hline Surgeon mean annual volume & $2.0(1.5,2.7)$ & $4.7(4.3,5.0)$ & $<.001$ & $0(0)$ \\
\hline Hospital mean annual volume & $8.7(6.0,13.2)$ & $10.2(8.7,17.8)$ & $<.001$ & $0(0)$ \\
\hline Elective procedure & $21(1.6)$ & $3(1.3)$ & $>.99$ & $0(0)$ \\
\hline Urgent procedure & $169(12.8)$ & $34(14.7)$ & .43 & $0(0)$ \\
\hline Emergency procedure & $1037(78.6)$ & $181(78.4)$ & .93 & $0(0)$ \\
\hline Salvage procedure & $92(7.0)$ & $13(5.6)$ & .45 & $0(0)$ \\
\hline Root segment & $438(33.2)$ & $82(35.5)$ & .50 & $0(0)$ \\
\hline Ascending segment & $1146(86.9)$ & $203(87.9)$ & .68 & $0(0)$ \\
\hline Arch segment & $152(11.5)$ & $46(19.9)$ & $<.001$ & $0(0)$ \\
\hline Concomitant $\mathrm{CABG}$ procedure & $171(13.0)$ & $30(13.0)$ & .99 & $30(1.9)$ \\
\hline Concomitant valve procedure & $521(39.5)$ & $99(42.9)$ & .34 & $29(1.9)$ \\
\hline Concomitant "other" cardiac procedure & $395(30.0)$ & $76(32.9)$ & .37 & $34(2.2)$ \\
\hline Cardiopulmonary bypass time, $\min$ & $196(152,259)$ & $197(154,254)$ & .86 & $44(2.8)$ \\
\hline Aortic cross-clamp time, $\min$ & $105(74,143)$ & $109(68,147)$ & .81 & $56(3.6)$ \\
\hline Circulatory arrest time, $\min$ & $29(20,39)$ & $20(15,31)$ & $<.001$ & $402(25.9)$ \\
\hline In-hospital mortality & $254(19.3)$ & $29(12.6)$ & .015 & $0(0)$ \\
\hline
\end{tabular}

Continuous variables shown as median (25th percentile, 75 th percentile); categorical variables shown as frequency (\%). CABG, Coronary artery bypass grafting.

of $25.1 \%$ in $2005 .{ }^{14}$ European registries in the United Kingdom and Germany have published operative mortalities of $23.1 \%$ and $17.0 \%$, respectively. ${ }^{15}$ A recent publication from Mount Sinai Medical Centre, using the Nationwide Inpatient Sample database of 5184 patients between 2003 and 2008, showed average operative mortality of $21.6 \% .{ }^{8}$ However, in the advent of this decade, there are multiple centers worldwide that are publishing in-hospital mortality rates for ATAD in the single digits. ${ }^{16-19}$
Throughout the United States, the model of aortic supercenters with high referral rates have existed for some time. It has been suggested that outcomes in thoracic aortic surgery could be improved nationwide in the United States if the acute care and emergency surgical treatment of most patients with ATAD were regionalized and restricted to institutions with high-volume multidisciplinary thoracic aortic surgery programs. ${ }^{20}$

Andersen et al. from the Duke group published their results pertaining to the impact model of a multidisciplinary

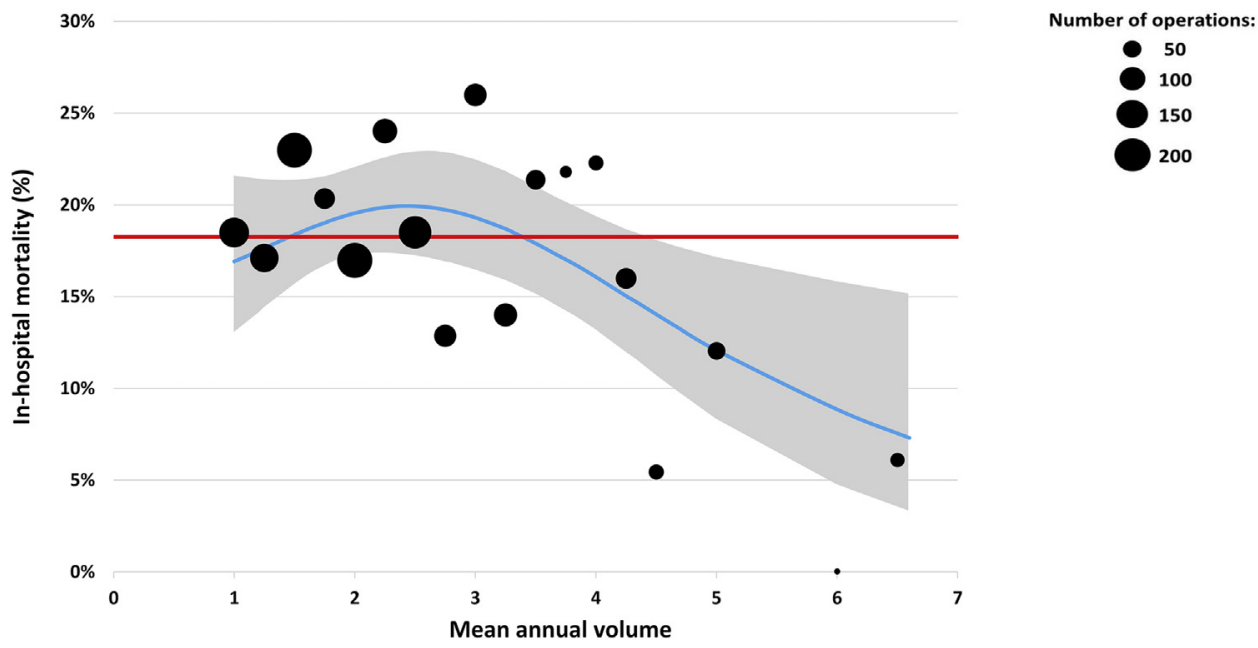

FIGURE 1. Trend in mean annual volume (MAV) of acute type A aortic dissection (ATAD) activity and observed mortality. Each black dot corresponds to the mean mortality (vertical axis) for ATAD procedures performed by consultant surgeons with a specific mean annual case volume (MAV) (horizontal axis). The size of the black dots is proportional to the total number of ATAD procedures performed by surgeons with the given MAV. Please note that, although volume was modeled continuously, to improve legibility the number of surgeon procedures is grouped for every 0.25 of a year; therefore, each dot can be composed of multiple consultant surgeons. The blue line is a fitted smoothing curve to illustrate the trend, adjusted for preoperative risk factors, and the grayshaded area denotes approximate $95 \%$ confidence intervals. The red horizontal line represents the overall mean observed in-hospital mortality (18.3\%) for the study cohort. 


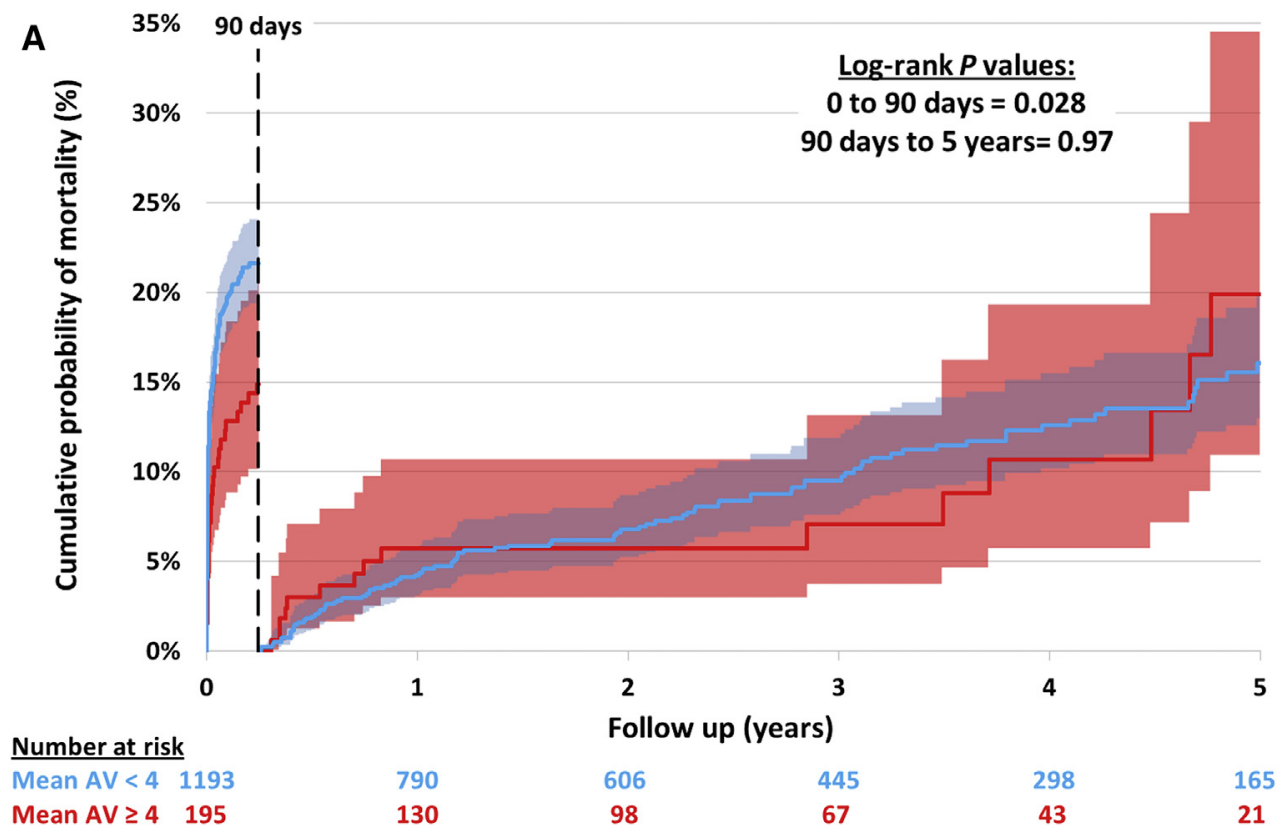

B

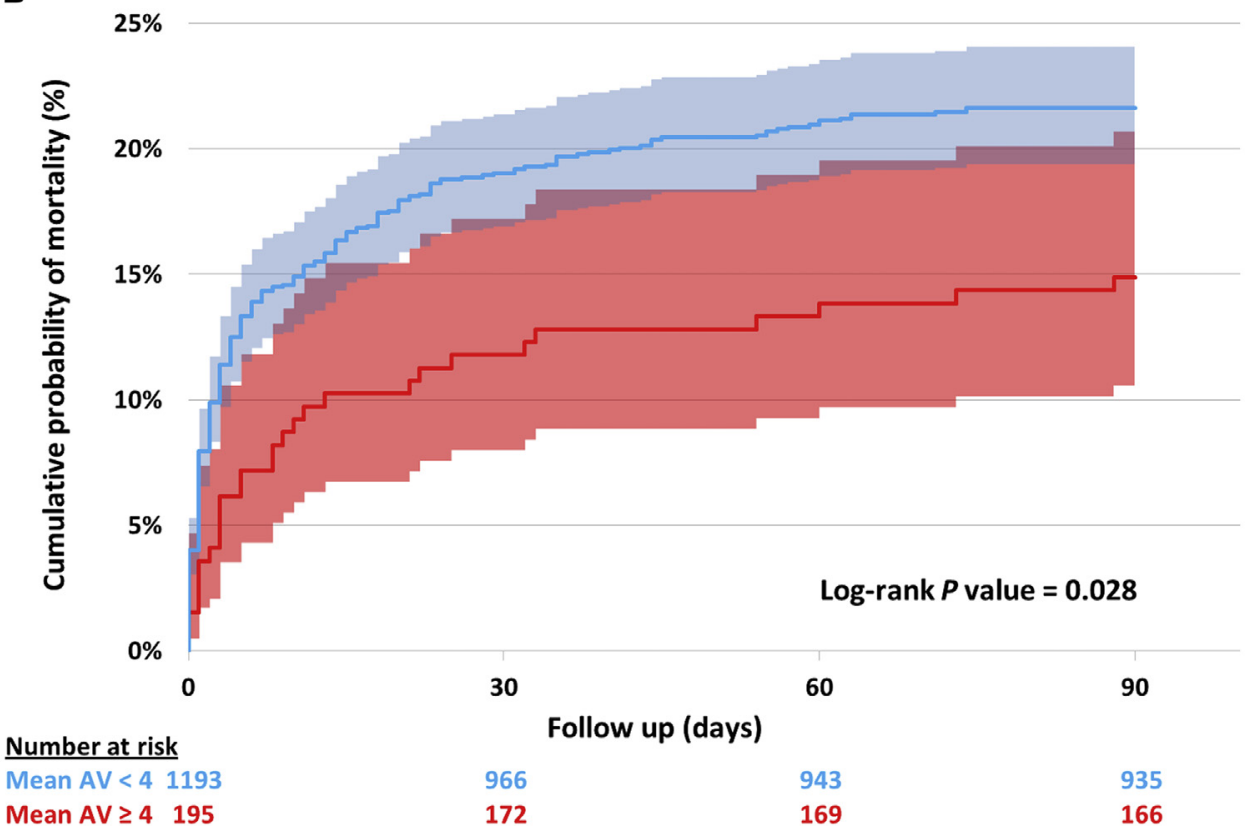

FIGURE 2. A, Kaplan-Meier chart showing the cumulative probability of all-cause mortality for acute type A aortic dissection (ATAD) patients in the surgeon mean annual case volume (MAV) groups. Landmark rebasing occurs at 90 days. Colored bands show approximate $95 \%$ confidence intervals. B, Kaplan-Meier chart showing the cumulative probability of all-cause mortality for ATAD patients in the surgeon MAV groups. Colored bands show approximate $95 \%$ confidence intervals ( 0 - to 90-day detail from Figure 2, $A$ ). AV, Annual volume.

team approach to ATAD. ${ }^{217}$ They reported operative mortality before multidisciplinary implementation was $33.9 \%$ and was statistically equivalent to the expected operative mortality rate of $26.0 \%$. Operative mortality after multidisciplinary implementation fell to $2.8 \%$ and was statistically better than the expected operative mortality rate of $18.2 \%$ using the IRAD preoperative prediction model.
In the United Kingdom, centralization of expertise and service provision for type A aortic dissection does not exist. Operating on ATAD does not generally follow a selective referral protocol, which in effect leads to a national mix and match between high- and low-volume surgeons. Compounding this is the lack of best practice evidence on structured referral from emergency room to operating room. This 
TABLE 3. Logistic regression analysis for in-hospital mortality

\begin{tabular}{lccc}
\hline \multicolumn{1}{c}{ Variable } & Odds ratio & $\mathbf{9 5 \%}$ confidence interval & P \\
\hline Surgeon mean annual volume & 0.853 & $0.733-0.992$ & .039 \\
Hospital mean annual volume & 1.005 & $0.956-1.057$ & .84 \\
Age at procedure, y & 1.028 & $1.015-1.041$ & \\
Previous cardiac surgery & 1.840 & $1.052-3.218$ & .001 \\
Peripheral vascular disease & 1.505 & $1.051-2.156$ & .033 \\
Left ventricular ejection fraction $<30 \%$ & 2.896 & $1.374-6.104$ & .026 \\
Preoperative cardiogenic shock & 1.722 & $1.137-2.607$ & .005 \\
Salvage procedure & 5.474 & $2.790-10.741$ & .010 \\
Concomitant CABG procedure & 2.135 & $1.412-3.229$ & $<.001$ \\
Cardiopulmonary bypass time, min & 1.003 & $1.002-1.005$ & $<.001$ \\
\hline
\end{tabular}

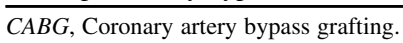

is due to multiple factors, which unfortunately have not been quantified in the United Kingdom. Those factors are in essence related to lack of swift recognition of ATAD. There is also lack of substantial evidence on the actual time to referral once ATAD is actually identified. The aforementioned mandate a policy that will serve better patient outcome and results across the United Kingdom.

Evidence of improved outcomes related to operative volume or surgeon expertise is often difficult to establish due to the infrequent nature of ATAD and consequent lack of statistical power that could potentially provide meaningful analysis. $^{21}$

The ideal definition of volume is inherently inconsistent; this makes diving into a discussion involving "volume" highlight caveats that are not potentially attained or addressed between cardiac surgical units at large. The strong rationale of the volume-outcome relationship as reflected in literature springs from the catalyst for subspecialization
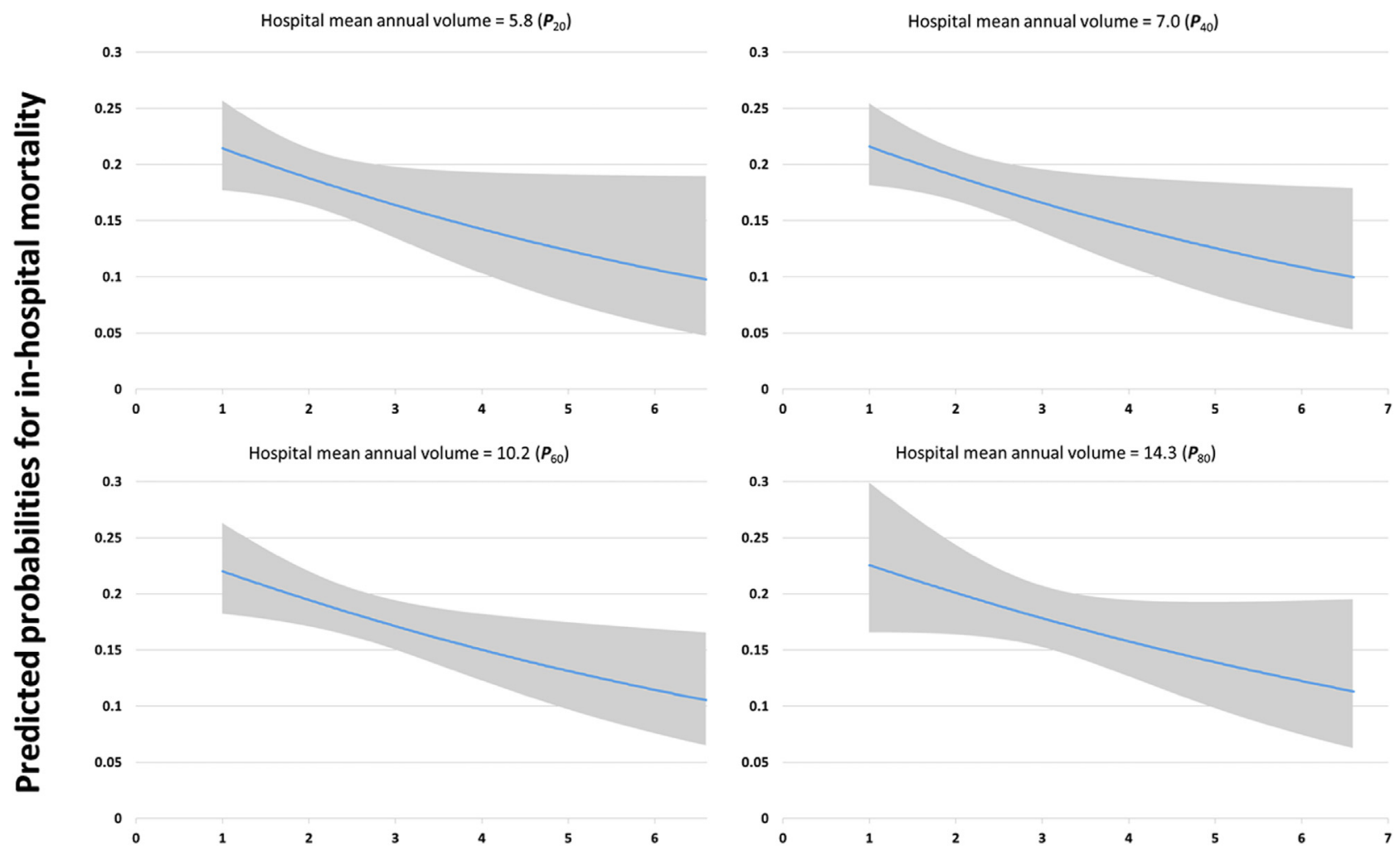

\section{Consultant mean annual volume}

FIGURE 3. Panel chart showing the interaction between hospital and surgeon volume. The blue lines in each chart represent predicted probabilities of inhospital mortality over the range of surgeon mean annual case volume (MAV) for the 20th, 40th, 60th, and 80th percentiles of hospital MAV, and the grayshaded areas denote approximate $95 \%$ confidence intervals. Overall $P$ value for interaction $=.88$. 
TABLE 4. Cox proportional hazards analysis for survival from procedure and from 90 days postprocedure

\begin{tabular}{|c|c|c|c|c|c|c|}
\hline \multirow[b]{2}{*}{ Variable } & \multicolumn{3}{|c|}{ Start time $=$ procedure date } & \multicolumn{3}{|c|}{ Start time $=90$ days postprocedure } \\
\hline & Hazard ratio & $\begin{array}{c}95 \% \text { confidence } \\
\text { interval }\end{array}$ & $\boldsymbol{P}$ & Hazard ratio & $\begin{array}{c}95 \% \text { confidence } \\
\text { interval }\end{array}$ & $\boldsymbol{P}$ \\
\hline Surgeon mean annual volume & 0.882 & $0.801-0.972$ & .011 & 0.920 & $0.779-1.088$ & .33 \\
\hline Hospital mean annual volume & 1.029 & $1.000-1.059$ & .050 & 1.020 & $0.972-1.072$ & .42 \\
\hline Age at procedure, $\mathrm{y}$ & 1.028 & $1.019-1.037$ & $<.001$ & 1.043 & $1.023-1.063$ & $<.001$ \\
\hline Left ventricular ejection fraction $<30 \%$ & 2.495 & $1.586-3.926$ & $<.001$ & 5.799 & $2.169-15.505$ & $<.001$ \\
\hline Preoperative cardiogenic shock & 1.426 & $1.068-1.903$ & .016 & 0.880 & $0.459-1.687$ & .70 \\
\hline Salvage procedure & 3.250 & $2.139-4.965$ & $<.001$ & 2.138 & $0.791-5.778$ & .13 \\
\hline Arch segment & 1.414 & $1.047-1.909$ & .024 & 1.200 & $0.653-2.207$ & .56 \\
\hline Concomitant $\mathrm{CABG}$ procedure & 1.629 & $1.235-2.150$ & $<.001$ & 1.215 & $0.636-2.323$ & .56 \\
\hline Cardiopulmonary bypass time, $\min$ & 1.003 & $1.002-1.004$ & $<.001$ & 1.001 & $0.998-1.003$ & .53 \\
\hline
\end{tabular}

$C A B G$, Coronary artery bypass grafting.

and centralization of aortic services. It is to provide centers with large and reaching catchment areas the reciprocal improvement and effect on the subspecialized unit. It allows more robust referral to influx and therefore maintain an adequate voluminous exposure. Essentially, in the United Kingdom, thoracic aortic aneurysm service is in much need of such an approach and a national policy and mandate that would support such programs across the United Kingdom should be warranted. This should provide a sustained increase in volume to concentrated expertise that would allow the possibility to address dire surgical diseases and avert associated complications. It will then reciprocate this arrangement by ultimately reducing mortality and perhaps improving survival and aftercare postsurgery. ${ }^{22} \mathrm{Be}-$ side this, the advent of technological superiority in diagnosis and surgical planning of aortic surgery and the understanding of the natural history is resulting in personalized and targeted therapies and surgical procedures to be done on a wider range of the affected population. This has allowed for such cost-effective diagnostic tests to be distributed to a smaller number of regional centers and for them to operate on this patient cohort. Hence, this has titrated the inexistence of specialist centers and diverted a large number of patients to be operated at local lower-volume institutions.

The development of standardization and subspecialization of acute aortic services requires a comprehensive assessment of the current status in aortic surgery in the United Kingdom. As such, our analysis has demonstrated that there is significant variation of in-hospital mortality around the country, with little relationship between volume and outcome at a hospital level. These results are contrary to those demonstrated by international groups, including the Mount Sinai group using the Nationwide Inpatient Sample of North America that reported that lower-volume surgeons and centers have approximately double the risk-adjusted mortality of patients undergoing repair by the highestvolume care providers. ${ }^{8}$

Our study does, however, demonstrate that higher individual surgeon volume was associated with lower in- hospital mortality. These relationships could be explained by a number of different factors such as case mix per individual surgeon, selection bias and variations in turn down practice, concentration of expertise to a particular surgeon within a hospital, and inadvertent subspecialization of a surgeon with interests to aortic interventions. On the other hand, and as demonstrated in the context of the analysis, the difference between low- and high-volume surgeons clearly points out the shorter aortic cross-clamp and cardiopulmonary time but increased circulatory arrest time in the low-volume group, which could be related to attempt of more frequent use or extended repair entailing arch segment replacement. Although this is not entirely understood, one way of scoping this further would be to look at this element within the cohort and run a thorough factor analysis.

Although this study demonstrates good overall mortality rates for ATAD in the United Kingdom, it is likely that further improvements could be achieved through the introduction of a quality improvement program for ATAD surgery. It is vital that such a program is implemented across the multidisciplinary thoracic aortic surgery team, including anesthesia, postoperative surgical intensive care, and operative perfusion specialists. Such a program also should involve standardized referral pathways and treatment protocols for ATAD repair. Another important contributing factor would be the development and implementation of a robust referral system and an initiative to hospital managers and commissioners for centralization of expertise in ATAD repair. This will reduce the waiting time and taxing of ATAD patients unnecessarily in acute services while diagnostics are being carried out.

\section{Limitations}

The main limitation of this study is its retrospective nature and the variable nature of data quality between institutions in the United Kingdom. There are also several confounding variables to consider that are not available in the NICOR dataset. The foremost of these factors is probably case selection: surgeons at tertiary referral centers 
are likely to operate on patients significantly longer after the acute event than local units; consequently, their surgical outcomes may benefit from both temporal patient selection (more stable patients are more likely to survive transfer), and more aggressive individual patient selection informed by the additional complications (such as malperfusion syndromes) that manifest hours to days after initial presentation. Other possible confounding variables include delays between diagnosis and intervention; referral bias and clustering; and presence, severity, and duration of end-organ ischemia.

\section{CONCLUSIONS}

Concentration of expertise and volume to appropriate surgeons who perform increasingly more complex aortic cases would be required to change the current paradigm of ATAD outcomes in the United Kingdom. Whenever feasible, ATAD repair should be performed by a highvolume surgeon to reduce operative mortality. It is reasonable to suggest a national standardization and quality improvement framework for ATAD treatment.

\section{Conflict of Interest Statement}

Authors have nothing to disclose with regard to commercial support.

The authors acknowledge all members of the Society for Cardiothoracic Surgery in Great Britain and Ireland who contribute data to the National Adult Cardiac Surgery Audit registry. The National Institute for Cardiovascular Outcomes Research, UCL, London, provided the data for this study.

Data Sharing: The United Kingdom National Adult Cardiac Surgery Audit registry is available to researchers on application to the National Institute of Cardiovascular Outcomes Research (NICOR), University College London. Full details on the NICOR data-sharing application process are available at https://www.ucl. ac.uk/nicor/access/application (last accessed December 22, 2016).

\section{References}

1. Olsson C, Thelin S, Stahle E, Ekbom A, Granath F. Thoracic aortic aneurysm and dissection: increasing prevalence and improved outcomes reported in a nationwide population-based study of more than 14000 cases from 1987 to 2002. Circulation. 2006;114:2611-8.

2. Meszaros I, Morocz J, Szlavi J, Schmidt J, Tornoci L, Nagy L, et al. Epidemiology and clinicopathology of aortic dissection. Chest. 2000;117:1271-8.

3. Bonser RS, Ranasinghe AM, Loubani M, Evans JD, Thalji NMA, Bachet JE, et al. Evidence, lack of evidence, controversy, and debate in the provision and performance of the surgery of acute type A aortic dissection. J Am Coll Cardiol. 2011;58:2455-74.
4. Ranasinghe AM, Strong D, Boland B, Bonser RS. Acute aortic dissection. BMJ. 2011:343:d4487.

5. Trimarchi S, Nienaber CA, Rampoldi V, Myrmel T, Suzuki T, Mehta RH, et al; on behalf of the International Registry of Acute Aortic Dissection Investigators. Contemporary results of surgery in acute type A aortic dissection: The International Registry of Acute Aortic Dissection experience. J Thorac Cardiovasc Surg. 2005;129:112-22.

6. Bridgewater B, Keogh B, Kinsman R, Walton P. Sixth National Adult Cardiac Surgical Database Report. London, England: Society for Cardiothoracic Surgery in UK and Ireland and Dendrite Clinical Systems Ltd; 2008.

7. Kruger T, Conzelmann LO, Bonser RS, Borger MA, Czerny M, Wildhirt S, et al. Acute aortic dissection type A. Br J Surg. 2012;99:1331-44.

8. Chikwe J, Cavallaro P, Itagaki S, Seigerman M, Diluozzo G, Adams DH. National outcomes in acute aortic dissection: influence of surgeon and institutional volume on operative mortality. Ann Thorac Surg. 2013;95: 1563-9.

9. Hickey GL, Grant SW, Cosgriff R, Dimarakis I, Pagano D, Kappetein AP, et al. Clinical registries: governance, management, analysis and applications. Eur J Cardiothorac Surg. 2013;44:605-14.

10. Bridgewater B, Keogh B, Kinsman R, Walton P. The Society for Cardiothoracic Surgery in Great Britain \& Ireland: The Sixth National Adult Cardiac Surgical Database Report. Henley-on-Thames, UK: Dendrite Clinical Systems Ltd; 2009.

11. Rosenbaum PR, Rubin DB. The central role of the propensity score in observational studies for causal effects. Biometrika. 1983;70:41-55.

12. Blackstone EH. Comparing apples and oranges. J Thorac Cardiovasc Surg. 2002; 123:8-15.

13. $2010 \mathrm{ACCF} / \mathrm{AHA} / \mathrm{AATS} / \mathrm{ACR} / \mathrm{ASA} / \mathrm{SCA} / \mathrm{SCAI} / \mathrm{SIR} / \mathrm{STS} / \mathrm{SVM}$ guidelines for the diagnosis and management of patients with thoracic aortic disease. Circulation. 2010;121:e266-369.

14. Tsai TT, Evangelista A, Nienaber C, Sechtem U, Fattori R, Myrmel T, et al. Long-term survival in patients presenting with type A aortic dissection: insights from the International Registry of Acute Aortic Dissection (IRAD). Circulation. 2005; 112:534-5.

15. Conzelmann LO, Krüger T, Hoffmann I, Rylski B, Easo J, Oezkur M, et al. German Registry for Acute Aortic Dissection Type A (GERAADA): initial results [in German]. Herz. 2011;36:513-24.

16. Sun L, Qi R, Zhu J, Liu Y, Chang Q, Zheng J. Repair of acute type A dissection: our experiences and results. Ann Thorac Surg. 2011;91:1147-52.

17. Andersen ND, Ganapathi AM, Hanna JM, Williams JB, Gaca JG, Hughes GC Outcomes of acute type a dissection repair before and after implementation of a multidisciplinary thoracic aortic surgery program. J Am Coll Cardiol. 2014; 63:1796-803

18. Vallabhajosyula P, Szeto WY, Pulsipher A, Desai N, Menon R, Moeller P, et al. Antegrade thoracic stent grafting during repair of acute Debakey type I dissection promotes distal aortic remodeling and reduces late open distal reoperation rate. $J$ Thorac Cardiovasc Surg. 2014;147:942-8.

19. Komatsu K, Takano T, Terasaki T, Wada Y, Seto T, Fukui D, et al. Surgical outcomes of acute type A aortic dissection in elderly patients. Ann Thorac Surg. 2014;97:1576-8.

20. Miller DC. Another meiosis in the specialty of cardiovascular and thoracic surgery: birth of the purebred "thoracic aortic surgeon"? J Am Coll Cardiol. 2014;63:1804-6.

21. Holt PJE, Poloniecki JD, Loftus IM, Thompson MM. The relationship between hospital case volume and outcome from carotid endarterectomy in England from 2000-2005. Eur J Vasc Endovasc Surg. 2007;34:645-54.

22. Appoo JJ, Pozeg Z. Strategies in the surgical treatment of type A aortic arch dissection. Ann Cardiothorac Surg. 2013;2:205-11.

Key Words: aorta, dissection, aneurysm, surgeon volumeoutcome, AAD 


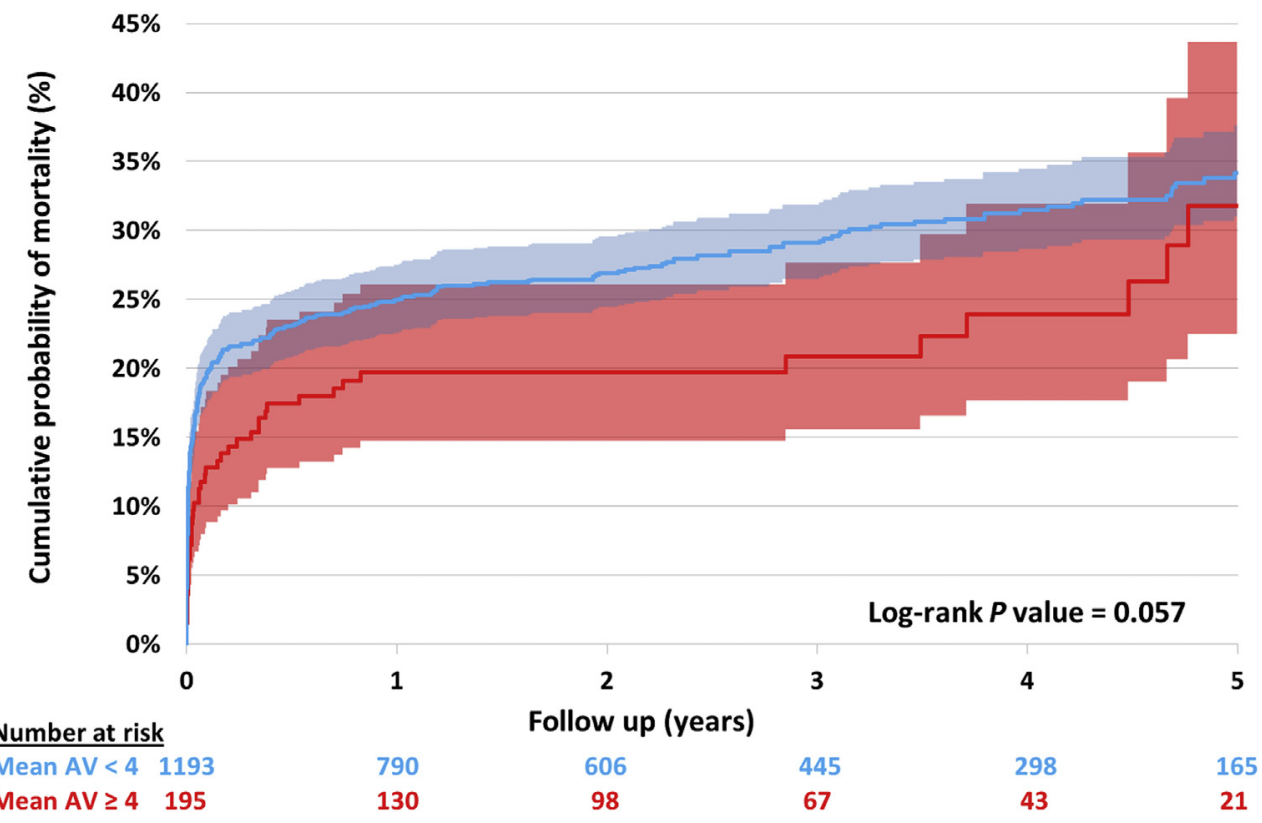

FIGURE E1. Kaplan-Meier chart showing the cumulative probability of all-cause mortality for acute type A aortic dissection patients in the surgeon mean annual case volume groups. Colored bands show approximate $95 \%$ confidence intervals. AV, Annual volume. 